\title{
Système rénine/angiotensine de l'utérus et de l'ovaire chez les femelles de Mammifères
}

\author{
S Le Gall, C Féral, P Leymarie
}

\author{
URA CNRS 609, laboratoire de Biochimie, CHU Caen, 14032 Caen Cedex
}

(Reçu le 14 décembre 1992; accepté le 23 février 1993)

\begin{abstract}
Résumé - Le système rénine-angiotensine (SRA) présent dans le sang des Mammifères contrôle la pression et la composition électrolytique du sang. L'enzyme rénine, synthétisée dans la cellule endocrine juxtaglomérulaire, initie dans le plasma la production du peptide actif, l'angiotensine II (AII). Tous les éléments du SRA sont présents dans l'ovaire et l'utérus des Mammifères. Pendant la gestation chez la femme, le taux de prorénine plasmatique augmente fortement. Son origine semble ovarienne au cours des premières semaines de grossesse et déciduale à partir du second trimestre. Au cours du cycle menstruel, la prorénine, la rénine et l'All sont produites en abondance par la thèque des follicules en phase préovulatoire. Le contrôle de cette synthèse par LH/hCG a été montré expérimentalement chez la lapine. La rénine ovarienne semble identique à la rénine rénale, mais elle demeure localisée dans les cellules de la thèque. Seule la prorénine inactive est sécrétée en abondance. La production intracellulaire de rénine thécale génératrice d'All suggère l'existence d'une fonction paracrine/autocrine du SRA ovarien. Des récepteurs à l'angiotensine, de type $A T_{2}$, ont été caractérisés au niveau des membranes cellulaires de la granulosa de ratte, où ils sont inhibés par $\mathrm{FSH}$ et dans la thèque de vache, où ils sont régulés positivement par LH. Outre son éventuelle participation à la néovascularisation du follicule, le SRA ovarien pourrait moduler la synthèse des stéroïdes sexuels en élevant le rapport androgènes/œstrogènes et participer ainsi à l'astrésie folliculaire.
\end{abstract}

rénine / angiotensine II / atrésie / ovaire / utérus

Summary - Ovarian and uterine renin-angiotensin system in mammals. The circulating reninangiotensin system (RAS) participates in the regulation of blood pressure and electrolyte metabolism. Renin, a proteolytic enzyme, synthesized in the kidney from its biological precursor, prorenin, cleaves its substrate angiotensinogen in the blood to form the active octapeptide, angiotensin II (AII). All the RAS components are present in the reproductive system of mammals. During pregnancy, the level of prorenin increases in the plasma. The ovary is the source of this prorenin during early pregnancy and maternal decidua later on. During the menstrual cycle, the thecal of preovulatory follicles synthesize prorenin, renin and All. Thecal renin systhesis is controlled by $L H / h C G$ as demonstrated in vivo and in vitro in the rabbit. Ovarian renin seems to be identical to kidney renin. Prorenin appears to be the major secretory product rather than renin, which remains intracellular. $A T_{2}$-type angiotensin II-receptors are expressed in the rat on follicular granulosa cells and could be downregulated by FSH. The bovine thecal cells also express $A T_{2}$-receptors, up-regulated by $L H$. These data are consistent with an autrocrine or paracrine role for ovarian RAS. It has been implicated in neovascularization of the follicle and regulation of steroidogenesis by increasing the androgen/ estrogen ratio, an index of follicular atresia. 


\section{INTRODUCTION}

Chez les Mammifères, le système rénineangiotensine (SRA) contrôle la pression sanguine et la composition électrolytique du sang via une cascade de réactions initiées par une enzyme rénale, la rénine, et aboutissant à la production d'un octapeptide actif, l'angiotensine II.

La rénine est une protéase acide de $45000 \mathrm{Da}$. Comme toutes les protéases acides, elle présente 2 courtes séquences homologues, à résidus aspartates, directement impliquées dans le site catalytique de l'enzyme.

La rénine existe sous deux formes : une rénine enzymatiquement active et une rénine inactive, correspondant au précurseur. Cette rénine inactive, ou "prorénine", peut être activée in vitro par clivage enzymatique; ainsi, la trypsine scinde le prosegment et découvre le site enzymatique actif.

En représentation tridimensionnelle, la rénine apparaît comme une protéine globulaire à 2 lobes symétriques ; le prosegment de la prorénine forme à la partie amino terminale une languette qui recouvre le site catalytique, inhibant ainsi l'activité enzymatique.

La rénine est la seule enzyme de la famille des protéases acides à circuler sous forme active dans le plasma; on ne lui connaît qu'un seul substrat, l'angiotensinogène, qu'elle hydrolyse à $\mathrm{pH}$ neutre, en angiotensine $\mathrm{I}$, transformée à son tour par l'enzyme de conversion en angiotensine II (All) (fig 1).

Véhiculée par le plasma et se fixant spécifiquement au niveau de la surrénale et des vaisseaux sanguins, l'All agit à distance et pourrait être considérée comme l'hormone du SRA. Mais contrairement aux hormones, elle est produite dans le plasma, par hydrolyse enzymatique.

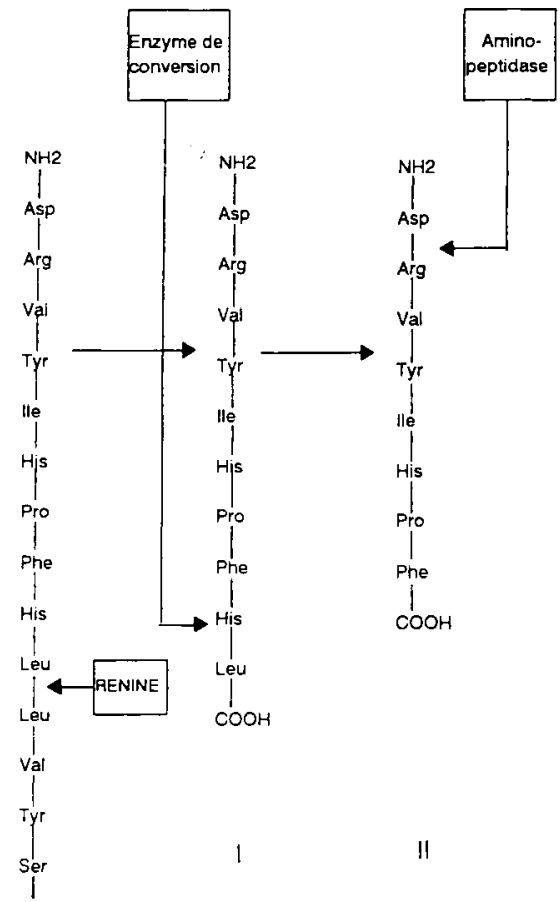

Angiotensinogène Angiotensines

Fig 1. Cascade métabolique initiée par la rénine.

À l'inverse, synthétisée comme une hormone par la cellule endocrine juxtaglomérulaire, la rénine agit en tant qu'enzyme dans le plasma (Corvol et Ménard, 1986).

Le précurseur inactif de la rénine, la prorénine, est essentiellement synthétisé dans le rein. Cependant, la sécrétion continue de prorénine dans le sang après binéphrectomie (Sealey et al, 1977) suggérait l'existence de sources tissulaires extrarénales.

Effectivement, la rénine a été identifiée par des techniques biochimiques et immunologiques au niveau de plusieurs tissus extrarénaux : vaisseaux sanguins, surrénale, foie, poumon, système nerveux, etc 
(Eskilden, 1973; Ganong, 1984; Naruse et al, 1985).

La rénine est également présente au niveau du système reproducteur femelle des Mammifères (Eskilden, 1973). Chez la femme, le taux de prorénine plasmatique est doublé au moment de l'ovulation et décuplé pendant la grossesse en parallèle avec la montée de hCG (Hsueh et al, 1982).

\section{LE SYSTĖME RÉNINE/ANGIOTENSINE DE L'UNITÉ UTÉROPLACENTAIRE}

Dès 1964, Gross et al ont mis en évidence une substance peptidique renin-like dans le placenta et l'utérus de lapine gestante. Chez la femme enceinte, on a pu montrer que l'unité utéro placentaire sécrétait de la prorénine : le taux de rénine inactive dans la veine utérine est significativement plus élevé que dans le sang périphérique (Brar et al, 1986).

Chez le cobaye, au cours de la gestation, les taux de rénine totale, de rénine active et d'All subissent une augmentation importante, aussi bien dans le placenta et dans le sang d'origine placentaire fœetale que dans le sang maternel systémique et utérin. En fin de gestation le chorion, le placenta et l'utérus présentent des concentrations tissulaires de rénine totale de l'ordre de 100 fois supérieures à celles du sang (Kalenga et al, 1991).

La prorérine utéroplacentaire est synthétisée par les cellules choriodéciduales (Symonds et al, 1968; Acker et al, 1982; Poisner et al, 1982; Pinet et al, 1988) et sécrétée dans le liquide amniotique, où on la trouve à forte concentration (Brown et al, 1964).

Une intéressante étude menée sur des membranes chorioniques pures intergémellaires a permis de préciser que la decidua utérine est la principale source de rénine utéroplacentaire (Shaw et al, 1989).
Cette origine déciduale explique la lenteur relative de la chute de la rénine post partum, comparée à la chute brutale de la hCG dont on connaît l'origine strictement placentaire (Sealey et al, 1985; 1986).

\section{LE SYSTĖME RÉNINE/ANGIOTENSINE OVARIEN}

\section{Mise en évidence d'un SRA ovarien}

Plusieurs observations faites chez les primates, et en particulier chez la femme, convergeaient en faveur d'une origine également ovarienne de la prorénine.

Chez la femme, il y a synchronisation entre les pics de LH et de prorénine circulantes aussi bien au cours du cycle menstruel normal (Sealey et al, 1987a) qu'au cours du cycle menstruel hyperstimulé lors d'un protocole de fécondation in vitro (Sealey et al, 1987b) (fig 2).

II existe une étroite corrélation entre le nombre de follicules préovulatoires obtenus et le taux de prorénine plasmatique mesuré au cours de cycles hyperstimulés (Itskovitz et al, 1987).

Dans le liquide folliculaire de la femme, on observe une forte concentration de prorénine (Fernandez et al, 1985a; Glorioso et al, 1986; Lightman et al, 1987a) et à un moindre degré de rénine active (Brameld et al, 1990) par rapport au plasma. L'All est également très augmentée (Culler et al, 1986).

Chez la femme, la prorénine et la rénine sont significativement plus élevées dans la veine ovarienne que dans le sang périphérique (Paulson et al, 1988).

De même, chez la femelle de babouin en milieu de cycle, on observe une élévation unilatérale du taux de prorénine dans le sang de la veine ovarienne drainant 

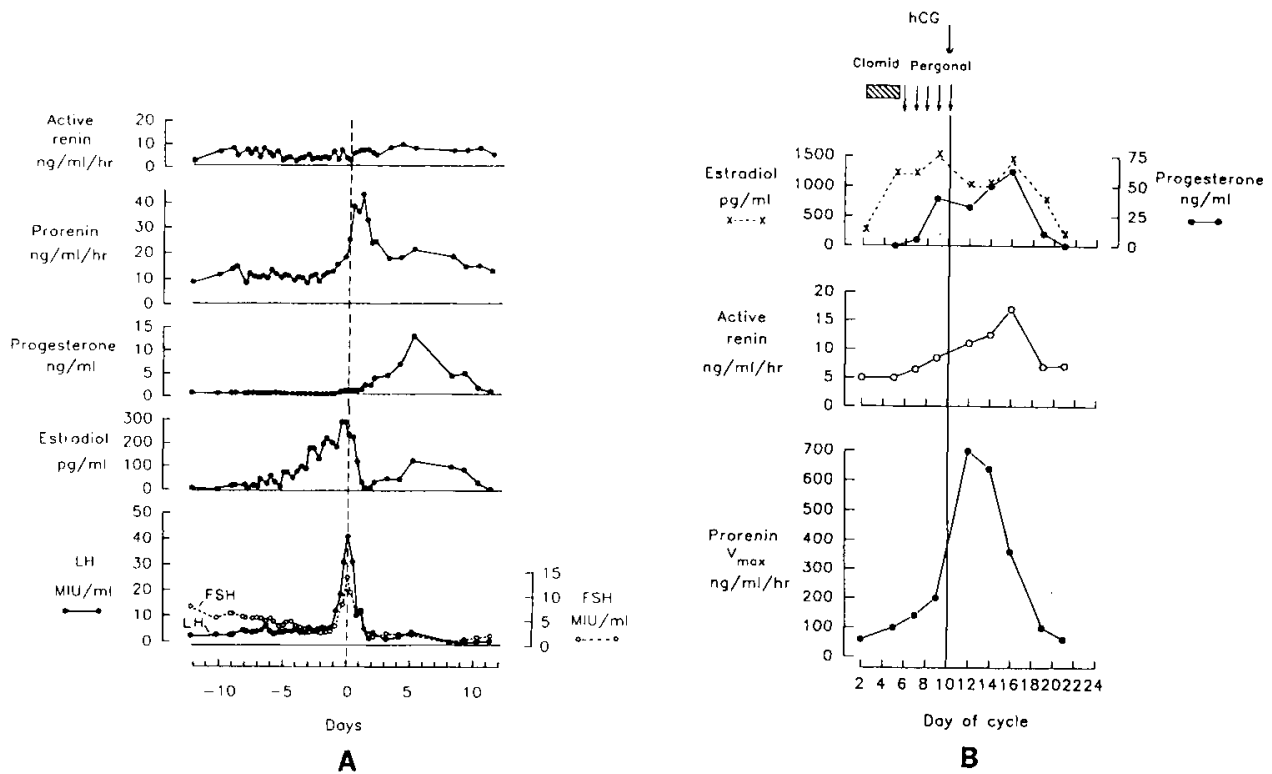

Fig 2. Évolution plasmatique des hormones sexuelles, de la prorénine et de la rénine au cours d'un cycle menstruel normal $(A)$ ou stimulé $(B)$ chez la femme (d'après Sealey et al, 1987a et b).

l'unique follicule préovulatoire. L'ovariectomie entraîne en 30 min la chute de ce taux de prorénine plasmatique (Sealey et al, 1990).

Chez une femme enceinte aux ovaires non fonctionnels mais ayant bénéficié d'un don d'embryon, il n'y a pas d'élévation du taux de prorénine circulante au cours du premier trimestre de la gestation (Sealey et al, 1987b; Derkx et al, 1987) (fig 3).

Tous les éléments du SRA sont présents dans l'ovaire : la rénine et son ARNm (Kim et al, 1987; Lightman et al, 1987b; Do et al, 1988; Palumbo et al, 1989; Schultze et al, 1989), l'angiotensinogène et son ARNm (Ohkubo et al, 1986), l'enzyme de conversion (Speth et al, 1988), I'All (Culler et al, 1986; Glorioso et al, 1986; Husain et al, 1987; Lightman et al, 1987a) et les récepteurs à l'All (Pucell et al, 1987 et 1988; Husain et al, 1987; Speth et al, 1986, 1988; BrunswingSpickenheier et Mukhopadhyay, 1992).
La convergence de ces éléments démontrait l'existence d'un SRA ovarien dont l'origine cellulaire, le mode de régulation et le rôle physiologique demeuraient hypothétiques.

\section{Origine cellulaire et régulation de la rénine ovarienne}

\section{SRA du follicule préovulatoire}

Chez la femme, l'isolement de cellules folliculaires a permis de mettre en évidence une sécrétion de prorénine par les cellules de la thèque et non par les cellules de la granulosa. L'étude en immunocytochimie a confirmé l'origine thécale de la prorénine (Do et al, 1988).

La rénine ovarienne n'étant sécrétée qu'au moment de l'ovulation, les études menées chez la femme sont entravées par la rareté du matériel humain et par la cycli- 

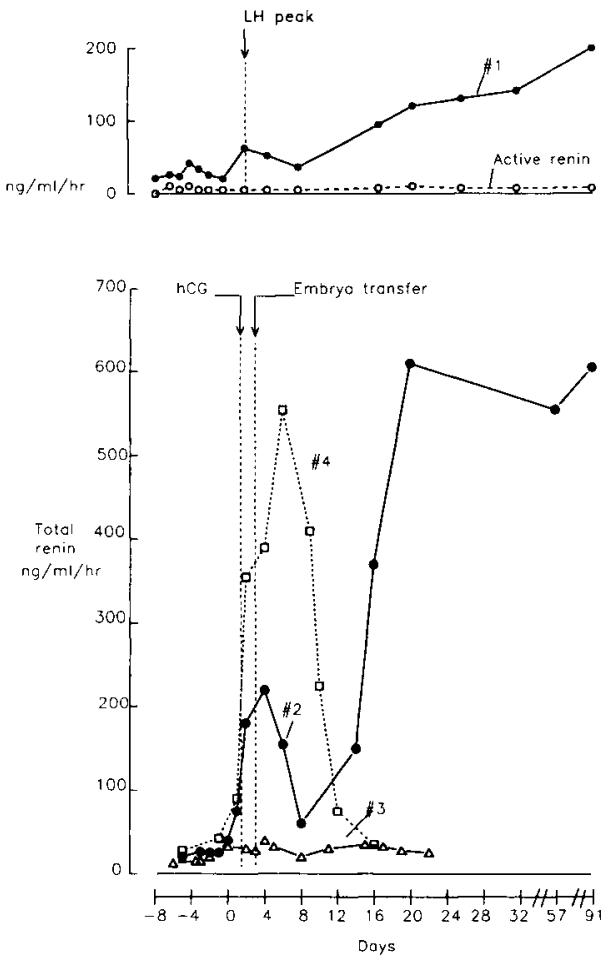

Fig 3. Évolution des taux de rénine plasmatique chez la femme : lors d'un cycle naturel suivi de grossesse (1), lors d'un cycle stimulé suivi de grossesse (2) ou non (4); cas d'une patiente avec ovaire déficient présentant une grossesse après don d'embryon (3) (d'après Sealey et al, $1987 b) . \bullet$ Rénine totale; $0 \longrightarrow$ rénine active.

cité de l'ovaire. En outre, dans les centres de fécondation in vitro, seules les cellules de granulosa sont récoltées avec les liquides folliculaires. Le prélèvement de cellules de thèque humaine n'est possible qu'à l'occasion d'ovariectomie.

En revanche, la lapine, ovulateur réflexe qui offre en permanence des follicules au stade préovulatoire, a permis d'explorer les mécanismes endocriniens qui contrôlent la production de rénine ovarienne. Ainsi, l'hyperstimulation par PMSG de lapines pré- pubères permet d'augmenter considérablement le nombre de follicules par ovaire (Féral et al, 1990). Dans ces follicules préovulatoires, la rénine totale intracellulaire est très abondante dans les cellules de thèque et à l'état de trace dans les cellules de granulosa. In vitro, la majeure partie de la rénine de la thèque reste intracellulaire, seulement $20 \%$ sont relargués dans le milieu. En revanche, la rénine est indétectable dans les cellules de granulosa et leur milieu de culture.

L'étude de l'expression in vivo du gène de la rénine dans l'ovaire de ratte semblait montrer l'existence d'une régulation par FSH (Kim et al, 1987).

Cependant, les données cliniques suggéraient que la sécrétion de rénine ovarienne était controlée par $\mathrm{LH} / \mathrm{hCG}$ : ainsi, la prorénine plasmatique augmente au cours de la grossesse en parallèle avec la montée de hCG, alors que le taux de FSH est très faible (Sealey et al, 1985).

L'existence d'une régulation par $\mathrm{LH} /$ hCG de la synthèse de la rénine ovarienne a été montrée chez la lapine (Féral et al, 1990). In vivo, une injection de LH/hCG entraine en $3 \mathrm{~h}$ une augmentation significative de la rénine totale intracellulaire dans la thèque, tandis qu'elie n'exerce aucune action au niveat: de la granulosa. $\mathrm{Pa}-$ rallèlement, la rénine plasmatique s'élève significativement. De même, après $24 \mathrm{~h}$ de culture en présence de hCG, la rénine totale intracellulaire de la thèque augmente de $400 \%$ par rapport au contrôle alors que le hCG est sans effet au niveau de la granulosa (fig 4).

Le fait que le taux de rénine intracellulaire de la thèque cultivée en absence de LH/hCG pendant $24 \mathrm{~h}$ ne diffère pas du taux de rénine endogène des cellules non cultivées prouve sans ambiguïté que l'augmentation induite in vitro par hCG correspond bien à une synthèse stimulable de rénine par la thèque (fig 4). 


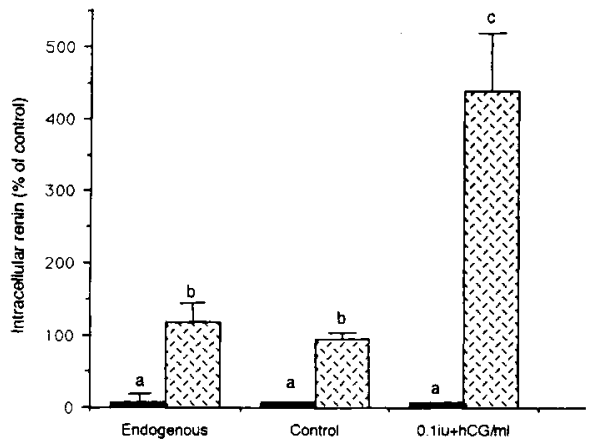

Fig 4. Stimulation in vitro par LH/hCG de la synthèse de rénine dans les cellules de thèque et de granulosa de lapine cultivées $24 \mathrm{~h}$. Le taux de rénine observé dans les cellules de thèque cultivées en l'absence de hCG est donné comme référence de base 100 . La rénine endogène est mesurée sur des cellules non incubées ( $n=4$ vs b et $b$ vs $c, P<0,01$ ) (d'après Féral et al, 1990). Granulosa; Theca.

\section{SRA du corps jaune}

La rénine a également été localisée dans le corps jaune des mammifères par dosage radio-immunologique chez le rat (Cabrera et al, 1986; de Vito et al, 1991) et par immunohistochimie chez la femme (Palumbo et al, 1989). L'ARNm de la rénine a été détecté par la technique d'hybridation in situ dans les cellules lutéales de rat (Lightman et al, 1987b).

\section{CARACTÉRISATION DE LA RÉNINE OVARIENNE}

\section{Rénine inactive et rénine active}

Selon la plupart des auteurs, la prorénine représente la forme majeure de la rénine produite par les tissus de l'ovaire et sécrétée dans le plasma (Sealey et al, 1987b), dans le liquide folliculaire (Schultze et al, 1989; Hageman et al, 1992) ou dans les milieux de culture de thèque (Do et al,
1988 et Paulson et al, 1989a). L'inactivité enzymatique de la prorénine pose dans ce cas le problème de la génération de l'All, présente à forte concentration dans le fluide follicuaire (Culier et al, 1986). Cette présence d'All implique nécessairement l'activation préalable de la prorénine inactive en une forme active.

La prorénine humaine a pu être partiellement activée in vitro par des micelles lipidiques de synthèse mimant la couche interne de la membrane cytoplasmique (Glorioso et al, 1989). Cette observation conforte l'hypothèse avancée par Sealey, selon laquelle le contact de la membrane pourrait entrainer in vivo une modification spatiale du prosegment permettant de démasquer le site enzymatique et d'activer ainsi la prorénine intracellulaire (Sealey et al, 1987b).

En revanche, d'autres travaux ont montré qu'une proportion importante de la rénine intracellulaire était constituée de rénine active : $75 \%$ de la rénine totale dans des homogénats d'ovaire de ratte (Howard et al, 1988), 50\% dans la thèque de follicule de vache (Schultze et al, 1989), et $45 \%$ dans l'utérus de lapine (Dzau et al, 1987).

Ces différences importantes observées dans la proportion des formes inactive/ active de rénine ovarienne pourraient être liées à la méthodologie du dosage. Dans la thèque du follicule de lapine les premiers dosages effectués dans notre laboratoire indiquaient seulement $5 \%$ de rénine active. Ultérieurement, il est apparu que deux décongélations successives détruisent largement l'activité rénine dans des échantillons conservés à $-80^{\circ} \mathrm{C}$ dans un tampon inhibiteur des protéases, alors que ce traitement reste sans effet sur les échantillons stockés dans du tampon PBS. Ainsi, si l'on évite les décongélations successives, les cellules de thèque de lapine renferment plus de $50 \%$ de la rénine totale sous forme active. 
Bien qu'on ignore la nature de l'enzyme qui pourrait être responsable in vivo de la maturation de la prorénine, on connaît plusieurs enzymes capables de scinder le prosegment : kallikréine, plasmine... (Dubin et al, 1991). L'augmentation importante de l'activité kallikréine observée dans l'ovaire de ratte en période périovulatoire (Espey et al, 1989) pourrait induire l'activation locale de prorérine à cette période. Quel que soit le mécanisme d'activation de la prorénine d'origine thécale, l'observation d'une production intracellulaire locale de rénine ovarienne active conforte l'hypothèse d'un SRA fonctionnel, générant de l'All active.

\section{Similitude des rénines ovarienne et rénale}

La rénine ovarienne de lapine est totalement inhibée in vitro par un antisérum dirigé contre la rénine de souris, cela exclut la possibilité d'une intervention au cours du dosage de la rénine d'une protéase non spécifique autre que la rénine. Les $\mathrm{pH}$ optima des activités enzymatiques de la rénine active et de la prorénine activée, déterminés dans les lysats de cellules folliculaires de lapine, se situent à pH 6,5. L'étude de la cinétique enzymatique montre que la valeur de la constante de Michaelis-Menten $(\mathrm{Km})$ pour la rénine ovarienne de la lapine $(1,7 \mu \mathrm{M})$ (Féral et al, 1990) est proche de celles déterminées par d'autres auteurs pour les rénines utérine $(1,8 \mu \mathrm{M})$ et rénale $(1,7 \mu \mathrm{M})$ de lapine (Dzau et al, 1987).

Des réactions immunologiques croisées ont été observées entre rénine du liquide folliculaire et rénine rénale chez la femme (Glorioso et al, 1986).

La similitude des paramètres optima d'activité enzymatique $(\mathrm{pH}, \mathrm{Km})$ et l'identité du comportement immunologique reflètent probablement une forte analogie voire une identité de structure des rénines provenant du rein et de l'ovaire, organes ayant une même origine embryologique.

\section{RÔLE PHYSIOLOGIQUE DES SYSTĖMES RÉNINE ANGIOTENSINE DE L'UTÉRUS ET DE L'OVAIRE}

L'existence d'un SRA localisé au niveau d'un tissu non impliqué dans la régulation de la pression sanguine pose le problème de son rôle physiologique.

Le système générateur d'All a été localisé dans la decidua utérine (Shaw et al, 1989) et dans la thèque du follicule préovulatoire (Féral et al, 1990) et l'All colocalisée avec la rénine par immunocytochimie au niveau de la thèque humaine (Lightman et al, 1986; Palumbo et al, 1989). La rénine synthétisée demeure essentiellement intracellulaire (Féral et al, 1990).

Des récepteurs à l'All ont été mis en évidence dans l'utérus (Capponi et Catt, 1979) et dans l'ovaire (Husain et al, 1987; Speth et Husain, 1988; BrunswingSpickenheier et Mukhopadhyay, 1992).

L'ensemble de ces éléments suggère l'existence, au niveau de ces 2 organes, d'une fonction paracrine ou autocrine de SRA intracellulaires.

\section{Rôle du SRA du chorion/decidua utérin}

Le SRA du chorion/décidua utérin pourrait réguler la circulation sanguine utéroplacentaire au cours de la gestation (Albertini et al, 1980). Cette action pourrait s'exercer via une modulation par l'All de la synthèse des prostaglandines (Ferris et Weir, 1983).

L'All qui provoque une néovascularisation des tissus (Fernandez et al, 1985b) et 
qui peut stimuler la division cellulaire, seule ou en potentialisant l'action mitogène de certains facteurs de croissance (dans Clauser et al, 1992), pourrait également participer à l'angiogenèse qui accompagne le développement de l'utérus chez la femelle gestante.

\section{Rôle du SRA ovarien}

Si une proportion importante de la rénine active est produite dans l'ovaire par la thèque des follicules prévulatoires, I'All libérée pourrait réguler localement la fonction folliculaire.

Les mécanismes subcellulaires impliqués dans la transduction de l'information véhiculée par l'All aux cellules ovariennes n'ont été que très partiellement élucidés.

Les récepteurs membranaires à l'All ont été caractérisés dans les cellules de granulosa de ratte hypophysectomisée et traitée au diéthylsilbestrol (Pucell et al, 1987). Spécifiques et saturables, ils présentent une haute affinité ( $\mathrm{Kd}=0,5 \mathrm{nM})$, leur densité est évaluée à 2000 sites de liaison par cellule. L'expression spontanée in vitro des récepteurs à l'All est régulée négativement en $48 \mathrm{~h}$ par FSH et All. L'action modulatrice de $\mathrm{FSH}$ sur les récepteurs à l'All s'exerce via l'AMPc tandis que l'inhibition par All de ses propres récepteurs passe par la voie du calcium (Pucell et al, 1988).

Les récepteurs à I'All présents dans les membranes de granulosa de rat sont de type $2\left(\mathrm{AT}_{2}\right)$ (Pucell et al, 1991). Les propriétés des recepteurs de ce type et notamment les mécanismes de signalisation transmembranaire et leurs fonctions sont encore mal connus, comparés à ceux des récepteurs $A T_{1}$, bien caractérisés dans la surrénale et les vaisseaux sanguins (Wong et al, 1992). La liaison de l'All aux récepteurs $A T_{2}$, contrairement à la liai- son aux recepteurs $A T_{1}$, n'entraîne pas d'inhibition de l'adénylate cyclase, agit indépendamment d'une protéine régulatrice liant le GTP, n'active pas la voie des phospho-inositides et n'augmente pas le calcium intracellulaire. De plus, dans la cellule de granulosa les récepteurs liés à l'All ne sont pas internalisés (Pucell et al, 1991).

Des récepteurs $\mathrm{AT}_{2}$ ont été localisés au niveau des membranes cellulaires de thèque de vache. $\mathrm{LH}$ exerce une régulation positive et dose-dépendante sur le nombre de ces recepteurs (Brunswig-Spickenheier et Mukhopadyay, 1992).

Les récepteurs $A T_{2}$ présents dans l'ovaire semblent largement distincts du point de vue fonctionnel des récepteurs $\mathrm{AT}_{1}$.

\section{Rôle angiogénique ou lutéolytique}

La participation de l'All à la néovascularisation du follicule au moment de la formation du corps jaune a été évoquée : on sait qu'il existe dans le liquide folliculaire un facteur angiogénique capable de stimuler la multiplication de cellules endothéliales (Frederick et al, 1985). La synthèse par les cellules lutérales de vache du FGF, à potentialités angiogéniques, est stimulable par l'All (Stirling et al, 1990).

D'autre part, dans ces cellules lutéales, l'All inhibe la sécrétion de progestérone stimulée par LH et parallèlement diminue la synthèse de l'ARNm codant pour le cytochrome $P_{450} s c c$ à l'origine du précurseur des stéroïdes (Stirling et al, 1990).

\section{Rôle dans l'ovulation}

Le rôle de l'All dans l'ovulation est controversé. Selon Pellicer et al (1988), l'All aurait un rôle direct dans l'ovulation chez le rat immature traité par PMSG et hCG; ré- 
sultat contesté par Daud et al (1989). Récemment, Yoshimura et al (1992) ont mis en évidence dans l'ovaire perfusé de lapine mature, un rôle facilitateur de l'All dans la rupture du follicule et la maturation de l'ovocyte.

\section{Rôle dans la synthèse des stéroïdes sexuels et dans l'atrésie folliculaire}

En se référant à la stimulation de la sécrétion d'aldostérone par l'All dans la corticosurrénale, les recherches ont principalement porté dans l'ovaire sur une éventuelle modulation de la synthèse des stéroïdes sexuels par l'All.

Dans le liquide folliculaire, les taux de prorénine et d'All évoluent parallèlement (Lightman et al, 1987a), ce qui suggère un rôle actif de ce peptide.

La localisation préférentielle des récepteurs à I'All au niveau des follicules atrétiques dans l'ovaire de ratte, alors que la densité de récepteurs à $\mathrm{FSH}$ sur ces mêmes follicules est faible, oriente vers une implication du SRA ovarien dans l'atrésie folliculaire (Husain et al, 1987; Daud et al, 1988).

Cette hypothèse est confortée par l'observation dans le liquide folliculaire de vache d'une relation inverse entre le taux d'œstradiol, considéré comme un marqueur de la croissance folliculaire (Hillier et al, 1980) et celui de la prorénine (Schultze et al, 1989 et Mukhopadhyay et al, 1991) (fig 5).

Dans des cellules de granulosa humaine isolées en culture, l'All stimule la sécrétion de testostérone et de progestérone, mais inhibe celle de l'œstradiol (Palumbo et al, 1988). Selon d'autres auteurs (Paulson et al, 1989b), l'All serait sans effet sur la sécrétion d'œstradiol par la granulosa humaine, mais stimulerait la production d'androstènedione par les cellules de thèque.

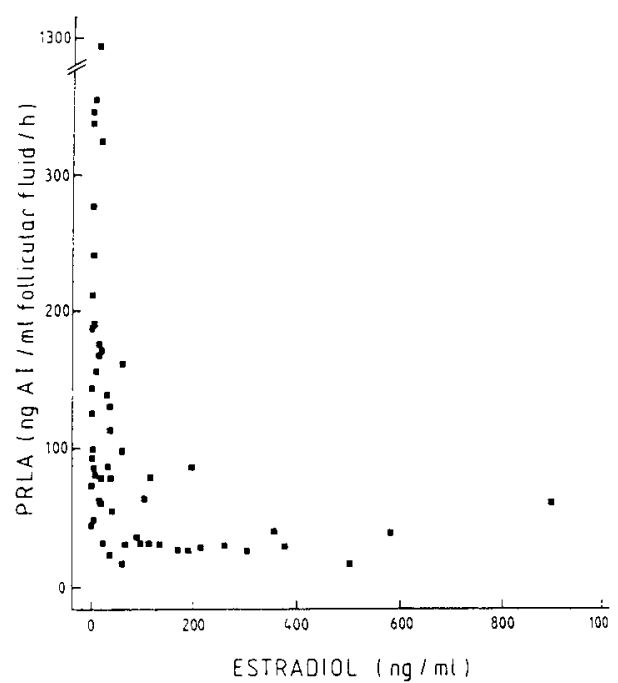

Fig 5. Corrélation inverse entre les taux de prorénine (PRLA : prorenin-like activity) et d'œstradiol dans le liquide folliculaire de la vache (d'après Schultze et al, 1989).

Des résultats également contradictoires ont été publiés pour le rat: in vitro, l'All stimule la sécrétion d'œstradiol et d'androgène par des fragments d'ovaire de ratte hyperstimulée par PMSG (Pucell et al, 1987; Bumpus et al, 1988).

Les résultats expérimentaux récents sur le hamster et le lapin confortent l'hypothèse d'un rôle de l'All dans l'atrésie folliculaire.

Dans les cellules de thèque de follicules de hamster, l'All augmente le nombre des gouttelettes lipidiques riches en ester de cholestérol, précurseur des stéroïdes. Parrallèlement, l'All élève significativement le rapport androstènedione $/ 17 \beta$ cstradiol sécrété par le follicule entier (Kitzman et Hutz, 1992) (fig 6), ce qui constitue un indice d'atrésie folliculaire (Carson et al, 1981).

Chez la lapine immature hyperstimulée par PMSG, les 2 types cellulaires du folli- 


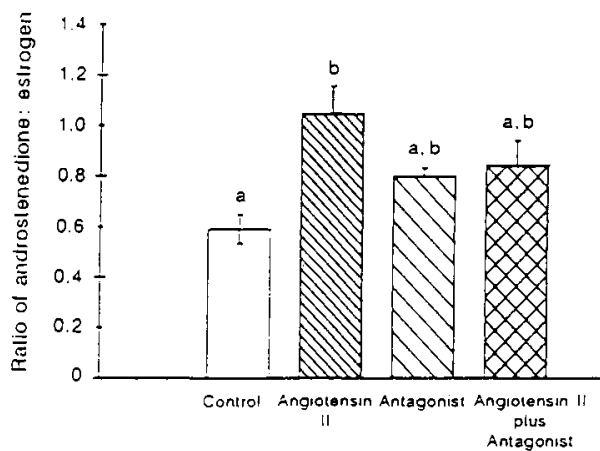

Fig 6. Influence de l'angiotensine II et de l'action d'un antagoniste (saralasine) sur le rapport androstènedione/œstradiol sécrétés par les follicules de hamster in vitro (d'après Kitzman et Hutz, 1992).

cule ovarien ont été séparés. In vitro, sous stimulation hCG/LH, l'All élève significativement le rapport androgènes de la thèque/œstrogènes de la granulosa et pourrait ainsi orienter le follicule vers l'atrésie folliculaire (Féral et al, en préparation).

On peut tenter d'intégrer ces données expérimentales et proposer un modèle de régulation de la croissance/atrésie du follicule par le SRA ovarien (fig 7).

Largement irrigué, le follicule dominant (fig 7A) fixe la LH sanguine au niveau des récepteurs membranaires des cellules de la thèque (Zeleznick et al, 1981). LH augmente la production de prorénine/rénine et All intracellulaires et l'expression des récepteurs à l'All de la thèque. L'All produite stimule alors la synthèse d'androgènes thécaux.

Les cellules de la granulosa du follicule dominant possèdent de nombreux récepteurs à FSH. Celle-ci exerce alors en quelques heures une inhibition de la synthèse des récepteurs à l'All. La granulosa du follicule dominant, dépourvue des récepteurs appropriés, pourrait ainsi échapper à l'action inhibitrice d'All et aromatiser activement les androgènes produits par la thè-

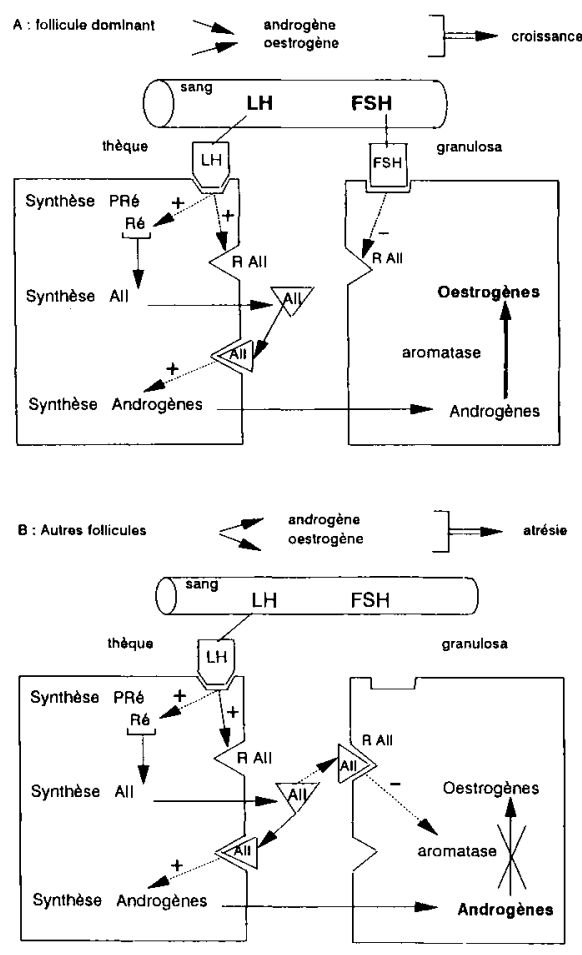

Fig 7. Schéma hypothétique illustrant le rôle du système rénine-angiotensine ovarien dans l'atrésie folliculaire. All : angiotensine II; PRé : prorénine; RAll : récepteur à l'angiotensine II; Ré : rénine.

que en œstrogènes qui amplifient la croissance du follicule dominant.

Par contre, l'irrigation des follicules voisins à croissance retardée (fig 7B) est médiocre. La FSH n'atteint leur granulosa qu'en faible quantité. En l'absence de $\mathrm{FSH}$, les récepteurs à l'All s'expriment alors spontanément au niveau des membranes des cellules de granulosa de ces follicules. L'All, sécrétée en abondance par la thèque, viendrait alors inhiber l'aromatase, et donc élever le rapport androgènes/ œstradiol, accélérant l'orientation de ces follicules vers l'atrésie. 
Ainsi, la localisation strictement intracellulaire de la production de prorénine/rénine dans l'ovaire protégerait l'organisme des éventuels effets pervers d'une production temporaire d'All tout en autorisant localement une action autocrine et paracrine sur le fonctionnement de l'ovaire.

\section{REMERCIEMENTS}

Nous remercions sincèrement Mme $C$ Marguerite pour sa participation à la préparation du manuscrit.

\section{RÉFÉRENCES}

Acker GM, Galen FX, Devaux C, Foote S, Papernik E, Pesty A, Menard J, Corvol P (1982) Human chorionic cells in primary culture: a model for renin biosynthesis. $J$ Clin Endocrinol Metab 55, 902-909

Albertini $R$, Seino $M$, Scichi $A G$, Carretero $O A$ (1980) Uteroplacental renin in regulation of blood pressure in the pregnant rabbit. $A m J$ Physiol 239, H266

Brameld JM, Broughton-Pipkin F, Symonds EM (1990) Studies on the human ovarian reninangiotensin system: optimization of assay methodology and effects of follicular stimulants. J Endocrinol 127, 513-521

Brar HS, Do YS, Tam HB, Valenzuela GJ, Murray $R D$, Longo $L D$, Yonekura $M L$, Hsueh WA (1986) Uteroplacental unit as a source of elevated circulating prorenin levels in normal pregnancy. Am J Obstet Gynecol 155, 12231226

Brown JJ, Davies DL, Doak PB, Lever AF, Robertson JIS (1964) The presence of renin in amniotic fluid. Lancet 2,64

Brunswig-Spickenheier B, Mukhopadhyay AK (1992) Characterization of angiotensin-ll receptor subtype on bovine thecal cells and its regulation by luteinizing hormone. Endocrinology 131, 1445-1452

Bumpus FM, Pucell AG, Daud Al, Husain A (1988) Angiotensin II: an intraovarian regulatory peptide. Am J Med Sci 295, 406-408
Cabrera RR, Guardia DC, de Vito E (1986) A renin-like enzyme in luteal tissue. Mol Cell Endocrinol 47, 269-273

Capponi AM, Catt KJ (1979) Angiotensin II receptors in adrenal cortex and uterus. $J$ Biol Chem 154, 5120

Carson RS, Findlay JK, Clark IJ, Burger HG (1981) Estradiol-17ß, testosterone, and androstenedione in ovine follicular fluid during growth and atresia of ovarian follicles. Biol Reprod 24, 105-113

Clauser E, Bihoreau C, Monnot C, Teutsch B, Conchon S, Davies E, Corvol P (1992) L'angiotensine $\mid 1$ est-elle un facteur de croissance ? Diabète Métab 18,129-136

Corvol P, Ménard J (1986) Du gène de la rénine aux inhibiteurs. Ann Endocrinol 47, 156-166

Culler MD, Tarlatzis BC, Lightman A, Fernandez $L A$, de Cherney AH, Negro-Vilar A, Naftolin $F$ (1986) Angiotensin II like immunoreactivity in human ovarian follicular fluid. J Clin Endocrinol Metab 62, 613-615

Daud A, Bumpus FM, Husain A (1988) Evidence for selective expression of angiotensin II receptors on atretic follicles in the rat ovary: an autoradiographic study. Endocrinology 122, 2727-2733

Daud A, Bumpus FM, Husain A, Naftolin F (1989) Angiotensin 11: does it have a direct obligate role in ovulation? Science 245, 870871

De Vito E, Guardia DC, Cabrera RR (1991) A renin-like enzyme in rat luteal tissue. Evidence of local synthesis and its regulation during pregnancy. J Endocrinology 128, 4350

Derkx FHM, Alberda AT, Zeilmaker GH, Schalekamp MAD (1987) High concentrations of immuno-reactive renin, prorenin and enzymatically active renin in human ovaries follicular fluid. Br J Obstet Gynecol 94, 4-9

Do YS, Sherrod A, Lobo RA, Paulson RJ, Shinagawat T, Chen J, Kjos S, Hsuet W (1988) Human ovarian theca cells are a source of renin. Proc Nat/ Acad Sci USA 85, 1957-1961

Dubin D, Pratt TE, Hui KY, Dzau VJ (1991) Characterization of prorerin activation using a synthetic peptide substract. J Hypertension $9,483-486$

Dzau VJ, Gonzalez D, Ellison K, Churchill S, Emmet $N$ (1987) Characterization of purified 
rabbit uterine renin: influence of pregnancy on uterine inactive renin. Endocrinology 120 , 358-362

Eskilden PC (1973) Renin in different tissues, amniotic fluid and plasma of pregnant and non pregnant rabbits. Acta Pathol Microbiol Scand $81 \mathrm{~A}, 263$

Espey LL, Tanaka N, Winn V, Okamura H (1989) Increase in ovarian kallikrein activity during ovulation in the gonadotrophin primed immature rat. $J$ Reprod Fertil 87, 503-508

Féral C, Resnik Y, Le Gall S, Mahoudeau J, Corvol P, Leymarie P (1990) Stimulation by hCG of ovarian inactive renin synthesis in rabbit preovulatory theca cells. $J$ Reprod Fertil 89, 407-414

Fernandez LA, Tarlatzis BC, Rzasa PJ, Caride VJ, Laufer N, Negro-Vilar AF, de Cherney AH, Naftolin F (1985a) Renin-like activity in ovarian follicular fluid. Fertil Steril 44, 219-223

Fernandez LA, Twickler J, Mead A (1985b) Neovascularization produced by angiotensin II. J Lab Clin Med 105, 141-145

Ferris TF, Weir EK (1983) Effect of captopril on uterine blood flow and prostaglandin $E$ synthesis in the pregnant rabbit. I Clin Invest 71,809

Frederick JL, Hoa NH, Preston DS, Frederick JJ, Campeau JD, Ono T, di Zerega GS (1985) Initiation of angiogenesis by porcine follicular fluid. Am J Obstet Gynecol 152, 1073-1078

Ganong WF (1984) The brain renin-angiotensin system. Annu Rev Physiol 46, 17-31

Glorioso N, Atlas SA, Laragh JH, Jewelewicz R, Sealey JE (1986) Prorenin in high concentrations in human ovarian follicular fluid. Science 233, 1422-1424

Glorioso N, Troffa C, Tonolo G, Manunta P, Maddedu P, Melis MG (1989) Activation of human prorenin by lipidic constituents of the cell membrane. Am J Hypertension 2, 920923

Gross F, Schaechtelin G, Ziegler M, Berger M (1964) A renin-like substance in the placenta and uterus of the rabbit. Lancet $\mathrm{i}, 914$

Hageman A, Nielsen AH, Dantzer V, Avery B, Poulsen K (1992) Measurement and identification of prorenin and renin in ovarian follicular fluid from cattle and pig. Clin Exp Pharmacol Physiol 19, 267-274
Hillier SG, van den Boogard AMJ, Reichert LE Jr, Van Hall EV (1980) Intraovarian sex steroid hormone interactions and the regulation of follicular maturation: aromatization of androgens by human granulosa cells in vitro. $J$ Clin Endocrinol Metab 50, 640

Howard RB, Pucell FM, Bumpus FM, Husain A (1988) Rat ovarian renin: characterization and changes during the estrous cycle. Endocrinology 123, 2331-2341

Hsueh WA, Luetscher JA, Carlson EF (1982) Changes in active and inactive renin throughout pregnancy. J Clin Endocrinol Metab 54, 1010-1016

Husain A, Bumpus FM, de Silva $P$, Speth RC (1987) Localization of angiotensin II receptors in ovarian follicles and the identification of angiotensin 11 in rat ovaries. Proc Natl Acad Sci USA 84, 2489-2493

Itskovitz J, Sealey JE, Glorioso N, Rosenwaks Z (1987) Plasma prorenin response to human chorionic gonadotropin in ovarian hyperstimulated women: correlation with the number of ovarian follicles and steroid hormone concentrations. Proc Natl Acad Sci USA 84, 7285

Kalenga MK, De Hertogh R, Whitebread S, Vankrieken L, Thomas K, De Gasparo M (1991) Étude du système rénine angiotensine et des stéroïdes chorioplacentaires, fœtaux et maternels chez le cobaye. J Physiol 85, 199-213

Kim SJ, Shinjo M, Tada M, Usuki S, Fukamizu A, Miyazaki H, Murakami K (1987) Ovarian renin gene expression is regulated by folliclestimulating hormone. Biochem Biophys Res Commun 146, 989-995

Kitzman PH, Hutz RJ (1992) In vitro effects of angiotensin II on steroid production by hamster ovarian follicles and on ultrastructure of the theca interna. Cell Tissue Res 268, 191196

Lightman A, Jones C, Fernandez LA, Boyers SP, de Cherney AH, Naftolin F (1986) The ovarian renin-angiotensin system: immunohistochemical localization of renin and angiotensin 11 (AII) immunoreactivity in human and rat ovary. In: Proc 68th annu meet Endocrine Soc. Anaheim, Californie (abstract $n^{\circ} 20$ )

Lightman A, Tarlatzis BC, Rzasa PJ, Culler MD, Caride VJ, Negro Vilar AF, Lennard D, de Cherney AH, Naftolin F (1987a) The ovarian renin-angiotensin system: renin-like activity and angiotensin $\mathrm{I} / \mathrm{I} \mathrm{I}$ immunoreactivity in go- 
nadotropin stimulated and unstimulated follicular fluid. Am J Obstet Gynecol 156, 808-818

Lightman A, Deschepper CF, Melon SH, Ganong WF, Naftolin F (1987b) In situ hybridization identities renin mRNA in the rat corpus luteum. Gynecol Endocrinol 1, 237

Mukhopadhyay AK, Holstein K, Szkudlinski M, Brunswig S, Picken-Heier B, Leidenberger FA, (1991) The relationship between prorenin levels in follicular fluid and follicular atresia in bovine ovaries. Endocrinology 129, 23672374

Naruse K, Murakoshi M, Osamura RY, Naruse $M$, Toma $H$, Watanabe $K$, Demura $H$, Inagami T, Shizume K (1985) Immunohistochemical evidence for renin in human endocrine tissues. J Clin Endocrinol Metab 61, 172-177

Ohkubo H, Nakayama k, Tanaka T, Nakanishi S (1986) Tissues distribution of rat angiotensinogen in RNA and structural analysis of its heterogeneity. J Biol Chem 261, 319-323

Palumbo A, Alam M, Lightman A, de Cherney AH, Naftolin F (1988) Angiotensin II affects in vitro steroidogenesis by human granulosa lutein cells. Proc Endocrinol Soc 1075, 289 (abstr)

Palumbo A, Jones $\mathrm{C}$, Lightman A, Carcangiu $M L$, de Cherney AH, Naftolin F (1989) Immunohistochemical localization of renin and angiotensin II in human ovaries. Am J Obstet Gynecol 160, 8-14

Paulson RJ, Do YS, Hsueh WA, Lobo RA (1988) Gradients of prorenin and active renin in ovarian venous and peripheral venous blood samples obtained simultaneously. Amer J Obstet Gynecol 159, 1575-1580

Paulson RJ, Eggena P, Do YS, Lobo RA, Hsueh WA (1989a) Ovarian renin production in vitro and in vivo. Characterization and clinical correlation. Fertil Steril 51, 634

Paulson RJ, Hernandez MF, Do YS, Hsueh WA, Lobo RA (1989b) Angiotensin II modulation of steroidogenensis by luteinized granulosa cells in vitro. Soc Gynecol invest (abstr \# 197)

Pellicer A, Palumbo A, de Cherney AH, Naftolin $F$ (1988) Blockage of ovulation by an angiotensin antagonist. Science 240, 1660

Pinet $F$, Corvol MT, Bourguignon J, Corvol $P$ (1988) Isolation and characterization of reninproducting human chorionic cells in culture. $J$ Clin Endocrinol Metab 67, 1211-1220
Poisner AM, Wood GW, Poisner R (1982) Release of inactive renin from human foetal membranes and isolate trophoblasts. Clin Exp Hypertens A4, 2007-2017

Pucell AG, Bumpus FM, Husain A (1987) Rat ovarian angiotensin II receptors. Characterization and coupling to estrogen secretion. J Biol Chem 262, 7076-7081

Pucell AG, Bumpus FM, Husain A (1988) Regulation of angiotensin II receptors in cultured rat ovarian granulosa cells by folliclestimulating hormone and angiotensin 11. J Biol Chem 263, 11954-11962

Pucell AG, Hodges JC, Sen I, Bumpus FM, Husain A (1991) Biochemical properties of the ovarian granulosa cell type 2- angiotensin II receptor. Endocrinology 128, 1947-1959

Schultze D, Brunswig B, Mukhopadhyay AK (1989) Renin and prorenin-like activities in bovine ovarian follicles. Endocrinology 124 , 1389-1399

Sealey JE, White RP, Laragh JH, Rubin AL (1977) Plasma prorenin and renin in anephric patients. Circ Res 41, 17-21

Sealey JE, Mc Cord D, Trufield PA, Ales KA, Drubin ML, Atlas SA, Laragh JG (1985) Plasma prorenin in first trimester pregnancy: relationship to changes in human chorionic gonadotrophin. Am J Obstet Gynecol 153, 514-519

Sealey JE, Glorioso MD, Itskovitz MD, Laragh $\mathrm{JH}$ (1986) Prorenin as a reproductive hormone. Am J Med 81, 1041-1046

Sealey JE, Cholst I, Glorioso N, Troffa C, Weintraub JD, James G, Laragh JH (1987a) Sequential changes in plasma $\mathrm{LH}$ and plasma prorenin during the menstrual cycle. J Clin Endocrinol Metab 65, 1-5

Sealey JE, Glorioso N, Itskovitz J, Atlas SA, Pitarresi TM, Preibisz JJ, Troffa C, Laragh JH (1987b) Ovarian prorenin. Clin Exp Hypertens Part A Theory Pract A9, 1435-1454

Sealey JE, Quimby FW, Itskovitz J, Rubattu S (1990) The ovarian renin-angiotensin system. Front Neuroendocrinol 11, 213-237

Shaw KJ, Do YS, Kjos S, Anderson PW, Shinagawa $T$, Dubeau L, Hsueh WA (1989) Human decidua is a major source of renin. J Clin Invest 83, 2085-2092

Speth RC, Bumpus FM, Husain A (1986) Identification of angiotensin II receptors in the rat ovary. Eur J Pharmacol 130, 351-352 
Speth RC, Husain A (1988) Distribution of angiotensin-converting enzyme and angiotensin II-receptor binding sites in the rat ovary. Biol Reprod 133, 695-702

Stirling D, Magness RR, Stone R, Waterman MR, Simpson ER (1990) Angiotensin II inhibits luteinizing hormone-stimulated cholesterol side chain cleavage expression and stimulates basic fibroblast growth factor espression in bovine luteal cells in primary culture. J Biol Chem 265, 5-8

Symonds EM, Stanley MA, Skinner SL (1968) Production of renin by in vitro cultures of human chorion and uterine muscle. Nature $217,1152-1153$
Wong PC, Chiu AT, Duncia JV, Herblin WF, Smith RD, Timmermans PB (1992) Angiotensin II receptor antagonists and receptor subtypes. Trends Endocrinol Metab 3, 211-217

Yoshimura $Y$, Karube M, Koyama N, Shiokawa S, Nannot T, Nakamura Y (1992) Angiotensin II directly induces follicle rupture and oocyte maturation in the rabbit. Febs Lett 307, 305308

Zeleznik AJ, Schuler HM, Reichert LE (1981) Gonadotropin-binding sites in the rhesus monkey ovary: role of the vasculature in the selective distribution of human chorionic gonadotropin to the preovulatory follicle. Endocrinology 109, 356-362 